\title{
Is there a correlation between biophotonical, biochemical, histological, and visual changes in the cartilage of osteoarthritic knee-joints?
}

\author{
Stephanie Tatjana Stumpfe ${ }^{1}$ \\ Julia Karin Pester ${ }^{1,2}$ \\ Susanne Steinert ${ }^{1,2}$ \\ Ivan Marintschev ${ }^{1}$ \\ Holger Plettenberg ${ }^{1,3}$ \\ Matthias Aurich ${ }^{1}$ \\ Gunther Olaf Hofmann ${ }^{1,2}$
}

1 Department of Traumatology, Hand and Reconstructive Surgery, Jena University Hospital, Jena, Germany

2 Department of Traumatology and Orthopaedic Surgery, Trauma Centre Halle (Saale), Jena, Germany

3 Arthrospec $\mathrm{GmbH}$, Jena, Germany

Corresponding author:

Gunther O. Hofmann

Department of Traumatology

Hand and Reconstructive Surgery

Jena University Hospital

Erlanger Allee, 101

07747 Jena, Germany

E-mail: gunther.hofmann@med.uni-jena.de

\section{Summary}

The aim of this study was to detect characteristic structural changes in the cartilage composition of osteoarthritis (OA), hereby improving the arthroscopic identification of cartilage pathology by the use of a non-destructive technique - NIRS (Near-Infrared Spectroscopy). 682 cartilage samples out of 25 knees with OA were classified visually, using the ICRS system, biophotonically, histologically $(n=66)$, using the Score of Mankin and the Score of Otte, and biochemically $(n=616)$, determining the content of glycosaminoglycan (GAG) and hydroxyproline (HP). Significant correlations were found between biophotonical, histological, biochemical and visual characteristics of cartilage lesions. NIRS values corresponded to the content of GAG, HP and to the Score of Mankin and Otte. The data show that changes in the composition and structure of articular cartilage influence the optical properties and can be measured objectively by NIRS. The ease of use during arthroscopy, the quick response and the non-destructive nature of NIRS make it a promising addition to the assessment of disease intervention in $\mathrm{OA}$.

KEY WORDS: cartilage, GAG, hydroxyproline, Mankin score, NIRS, osteoarthritis.

\section{Introduction}

$\mathrm{OA}$ is the most common joint disorder worldwide, being prevalent in the elderly, but also affecting younger people ${ }^{1}$. However, the pathogenesis of $O A$ is a multifactorial process and involves the whole joint and soft tissue structures in and around the joint ${ }^{2}$.

OA causes several changes in cartilage and leads to loss of tissue integrity and function. The breakdown of the extra cellular matrix (ECM) is most prominent and is indicated through the loss of the major matrix molecules, including proteoglycan and collagen ${ }^{3}$. This matrix transformation occurs although the chondrocytes are thought to have anabolic activity, such as matrix synthesis and proliferation. The cells seem to counteract the matrix degradation during the degenerative events of $\mathrm{OA}^{4,5}$. The biomechanical, biochemical, and histological properties of articular cartilage change as a consequence of cartilage degeneration in $O A^{6,7}$. Structural changes result in a break-up of the collagen-proteoglycan matrix with consequent changes in the water-binding properties and a shift in the composition of main cartilage components ${ }^{8,9}$.

There is increasing research interest in the physiological and pathological function of vital articular cartilage depending on the structure, the composition and the integrity of the tissue, carried out by correlating results of biophysical, biomechanical, biochemical, and histological methods. These methods proved as reliable and were validated in the past. However, nearly all of the methods in cartilage research are tissue-destructive techniques effecting the cartilage samples and are not applicable in joint preserving surgery.

NIRS is a non-destructive optical technique in the range of $780 \mathrm{~nm}$ up to $2500 \mathrm{~nm}$ and widely employed in many fields of medicine ${ }^{10,11}$. With this biophotonic measurement procedure it is possible to detect the overtone and combined vibration of for example $\mathrm{C}-\mathrm{H}$, $\mathrm{O}-\mathrm{H}$ und $\mathrm{N}-\mathrm{H}$ bondings. Therefore it is possible to get the link with this biophotonic measurement method to the chemical and biomechanical properties of analysed cartilage. Recent studies have shown that NIRS is a proven sensitive and accurate diagnostic in-vivo method for quantitative determination of structural and mechanical changes in cartilage ${ }^{12-14}$. Since infrared light interacts with cartilage components affected by OA, such as water, water-binding properties, $\mathrm{CH}-\mathrm{OH}$-and $\mathrm{SH}$-groups, early stage $\mathrm{OA}$ are detectable with this method ${ }^{12,13}$. Still, the rationale of this is not clearly understood.

The topic of this current study was to prove whether NIRS is able to identify structural and biochemical 
changes in human cartilage of $\mathrm{OA}$ and to evaluate the possible correlation between cartilage breakdown and loss of histological structure with biophotonical values. We hypothesized that NIRS is sufficient for the prediction of these processes of intra-cartilaginous degeneration.

\section{Methods}

\section{Patients}

Twenty-five knee-joints in 24 patients (13 women, 11 men) with severe osteoarthritis (OA) due to clinical $(\mathrm{KOOS})^{15,16}$ and radiological (Kellgren-Lawrence) $)^{17}$ scores received total knee replacement (TKR) with patella resurfacing and were enclosed in this prospective, single-centre, open-label study between March, 2009 and December, 2009. Mean age of the patients was 68.71 years (women 71.62 years, men 64.18 years), ranging from 52 to 80 years. All implantations were indicated by clinical (pain, loss of function and mobility) and radiological signs of OA. Informed consent was obtained from all patients after the nature of all examinations had been fully explained, following written approval from the local Ethics Committee for Clinical Trials (1714-01/06). All procedures were in accordance with the Helsinki Declaration concerning human trials.

\section{Surgery}

All patients received TKR under general anaesthesia, performed by two experienced surgeons. The cartilage in the femorotibial and femoropatellar compartments was independently examined by them using the classification approved by the International Cartilage Repair Society (ICRS) ${ }^{18}$. Joint surfaces were resected under continuous irrigation with Ringer's solution for cooling, covered with PBS-soaked gauze and immediately transferred to the laboratory for further investigations. Only cartilage samples of ICRS grades 1 - 3 were processed further in this study.

\section{Sample preparation}

Full-thickness osteochondral cylinders $(n=682)$ including subchondral bone with a diameter of $4 \mathrm{~mm}$ were drilled out of three different surface areas (femoral, tibial and patellar) using a gouge bit under continuous irrigation with Ringer's solution for cooling. The samples represented various stages of cartilage damage (7 ICRS grade 0; 360 grade 1; 190 grade 2; and 125 grade 3 ). Articular compartments with ICRS grade 4 were excluded from further investigation. The drilled-out specimens were initially examined biophotonically and then stored in acetone (Roth, Karlsruhe, Germany) for the further histological preparation or frozen for the biochemical examination.

\section{Biophotonical testing}

All samples ( $n=682$ ) were examined by a prototype NIRS device for diffuse reflectance measurement (arthrospec ONE, arthrospec $\mathrm{GmbH}$, Jena, Germany). The system consists of a photodiode array spectrometer (950 nm - 1700nm, 256 pixel), a stabilized broadband light source, a fibre-optic reflectance probe and associated hard and software for data acquisition (Fig. 1). For measurement, the face of the probe was placed on the cartilage surface of each specimen and five subsequent reflectance spectra were recorded (100 ms recording time per spectrum). To search for fundamental relations between laboratory data (presented as single value vectors) and optical data (matrix of spectral data) we used a multivariate regression model (partial least square regression) to calculate linear correlations. To avoid overfitting, we restricted the number of latent factors to five. The goodness of fit is described by the correlation coefficient between the best model and the laboratory data. A schematic diagram of the test setup is given in Figure 2.

Due to the exclusion of articular compartments with ICRS grade 4, there were different numbers of samples of each knee available which were examined by NIRS and processed during the following procedures. After the biophotonical testing, the specimens were divided into two groups: the first group concluded specimen of each patient with each ICRS grade for histological evaluation ( $n=66)$. The second group concluded remaining samples for biochemical analysis $(n=616)$. Criterion for the following selection of samples was the intention to harvest material from each patient with each ICRS grade for histological evaluation. All other specimens served for biochemical analysis.

\section{Biochemical Testing}

A total of 616 cartilage samples were shredded and incubated in papain working solution $(125 \mu \mathrm{g} / \mathrm{ml})$ for $16 \mathrm{~h}$ at $60^{\circ} \mathrm{C}$ after evaluation of the dry weight (Ohaus Europe GmbH, Nänikon, Switzerland).

\section{GAG content}

GAG content of these prepared samples was evaluated using the dimethyl-methylene blue (DMMB) assay. GAGs are a covalently attached functional group to the core proteins of proteoglycans. Aggrecan has a high affinity for water by virtue of its high negative fixedcharge density and causes the interaction with the $\mathrm{H}_{2} \mathrm{O}$-based DMMB reagent (Sigma-Aldrich, Steinheim, Germany). Therefore, the maximum absorption is changed and can be evaluated with a special reader (Sunrise, Tecan, Switzerland) at a wavelength of $\lambda=$ $525 \mathrm{~nm}$. The samples were diluted three times (1:200, $1: 400,1: 800)$ with dilution buffer $(0.05 \mathrm{M}$ Na-acetate and $0.05 \%$ Tween 20 ) and the results were averaged to get reliable information about the GAG content of 
Is there a correlation between biophotonical, biochemical, histological, and visual changes in the cartilage of osteoarthritic knee-joints?
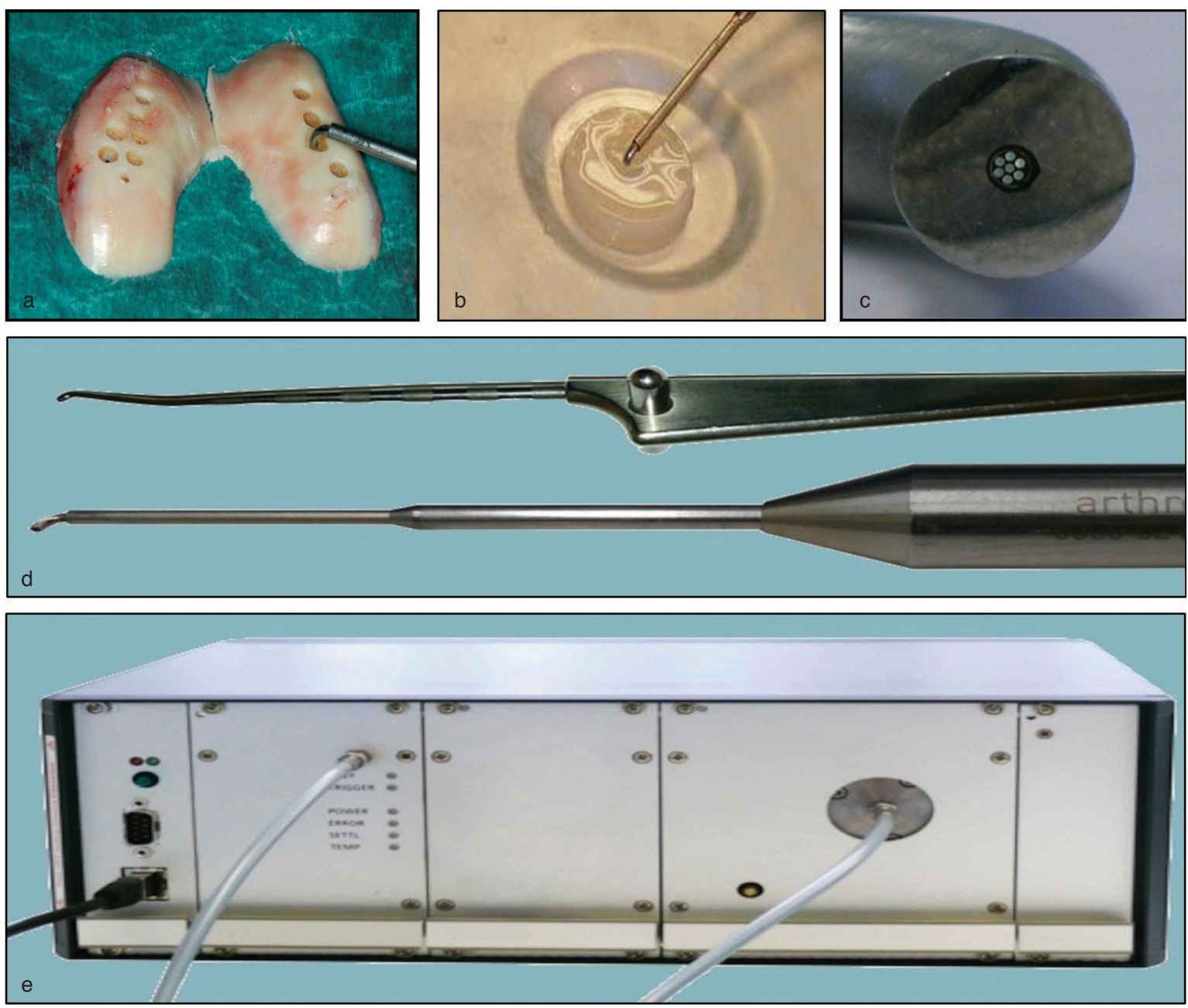

Figure 1. Design of biophotonic test setup. Full-thickness osteochondral cylinders from femoral surface area (a) were analysed by a prototype NIRS device (c-e). For measurement, the face of the probe was placed on the cartilage surface of each specimen $(a, b)$. The system consists of a photodiode array spectrometer (e), a stabilized broadband light source, a fibre-optic reflectance probe (c, d) and associated hard and software for data acquisition.

each sample. For each passage a standard curve was made. The results are reported as the mean $\pm S D$ of the analysis of three different diluted cultures.

\section{HP content}

All chemicals were purchased from Sigma Aldrich (Steinheim, Deutschland) or Thermo Fisher (Waltam, USA) unless otherwise noted. Determination of HP was performed by a colorimetric assay after papain digestion and freeze-drying. Aliquots from each digest were hydrolysed in $4 \mathrm{~N} \mathrm{NaOH}$ for 30 min at $125^{\circ} \mathrm{C}$. The hydrolysates ( $\mathrm{ph}=6.0$ ) were incubated for $20 \mathrm{~min}$ at room temperature with chloramine-T and afterwards with aldehyde-perchloric acid for $20 \mathrm{~min}$ at $70^{\circ} \mathrm{C}$ for colorimetric reaction. The absorbance at 550 $\mathrm{nm}$ was determined with a spectrophotometer (Sunrise, Tecan, Switzerland). The content of HP in each sample was calculated after generation of a standard curve using known concentrations of gelatin. The cal- culated HP content was used to determine the total amount of collagen by multiplying the results by a factor of 8.2. The results are reported as the mean \pm SD of the analysis of three separate cultures.

\section{Histological examination}

\section{Sample embedding}

The embedding of the specimens was carried out using Technovit 9100 (Heraeus Kulzer, Wehrheim/Ts., Germany) according to the manufacturers' protocol. This polymerisation system is based on methylmethacrylate (MMA) that hardens at low temperatures and has been developed especially for the embedding of mineralised tissues.

The embedded samples were cut into $4 \mu \mathrm{m}$ sections using a Leica microtome (Polycut S Reichert-Jung, Bensheim, Germany). Each sample was stained histologically for grading according to Mankin ${ }^{19}$ and Otte 20 . 


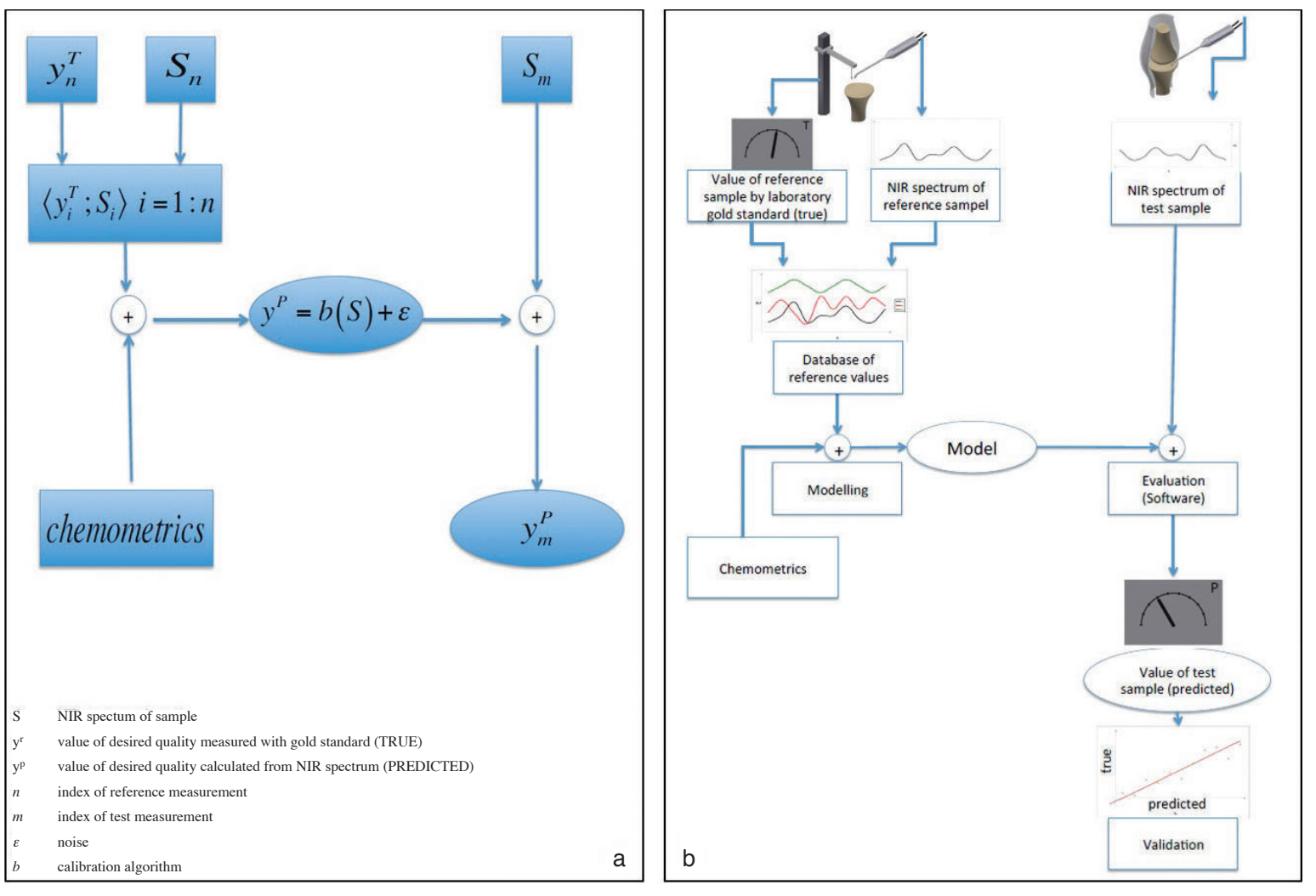

Figure 2. Schematic diagram of biophotonic test setup, validation and evaluation. (a) Model and formula of calculating the dependent variable from a NIR spectrum. (b) Typical steps of NIRS measurement. To create a model algorithm a set of reference data has to be recorded and analysed by chemometric tools.

\section{Microscopic staging}

Cartilage samples were staged microscopically according to the modified Mankin score ${ }^{19}$, which is estimated to be the actual gold standard scale concerning the evaluation of cartilage degeneration. The sections were stained with Hematoxylin-Eosin and Safranin-O and investigated microscopically. The four different aspects of the score were determined and summed up leading to a scale that ranges between 0 and 14 . The Mankin score 0-2 represents normal cartilage, 3-5 superficial fibrillation, 6-7 - moderate cartilage destruction, 8-10 - severe damage of cartilage, and over 10 - complete loss of cartilage. In addition, cartilage samples were classified microscopically according to the Otte score 20 . Chondral lesions were subdivided into four grades: Grade 0 represents intact normal cartilage, grade 1 - superficial fibrillation, grade 2 - chondral lesions (fissures and cracks) without extending down to subchondral bone, grade 3 - chondral lesions extending down to subchondral bone, and grade 4 focal or general full-thickness cartilage loss.

\section{Statistical Analysis}

We used PASW Statistics (PASW Statistics 17, SPSS Inc., Chicago, USA) for statistical analysis and Microsoft Excel (Microsoft Office Excel 2003, Mi- crosoft Corp., Redmond, USA) for data visualization. The data are presented as means, whereas we used normalised data to evaluate the relative change between the consecutive measurements.

For the establishment of the biophotonical correlation analysis all samples deemed as independent variable because bivariate correlations between several samples and six methods were performed. All other statistical analyses were made assuming that the variables were dependent. Normal distribution was tested with the Kolmogorow-Smirnow test. In case of normal distribution, we employed the t-test; otherwise, we used the Wilcoxon test. When parameters showed no normal or linear distribution, Spearman's rank correlation coefficient $\rho$ was used to characterise links between parameters. Significance was defined as $P \leq 0.05$.

Samples of the small group of ICRS grade 0 were excluded for statistical evaluation.

\section{Results}

\section{Macroscopic visual evaluation}

The samples represented various stages of cartilage damage, and cartilage abrasion was evident in most specimens. Only a small number of classified samples $(n=7)$ showed no OA signs (ICRS grade $=0$ ), 360 samples showed mild OA signs (ICRS grade = 
Is there a correlation between biophotonical, biochemical, histological, and visual changes in the cartilage of osteoarthritic knee-joints?

1), 190 samples showed grade 2 associated alterations, and 125 samples showed advanced OA signs (ICRS grade $=3$ ).

\section{Biochemical evaluation}

The group includes a total of 616 samples, consisting of six samples of ICRS grade 0, 338 samples of ICRS grade 1, 167 samples of ICRS grade 2, and 105 samples of ICRS grade 3 . The results are summarized in Table 1 and visualized in Figure 3. The average content of GAG was $35.62 \mu \mathrm{g} / \mathrm{mg}$ (SD: $14.15 \mu \mathrm{g} / \mathrm{mg}$ ) and of HP $96.34 \mu \mathrm{g} / \mathrm{mg}$ (SD: $49.78 \mu \mathrm{g} / \mathrm{mg}$ ). If considered separately according to the ICRS grades, the content of GAG and HP showed no significant differences in the mean values. This is reflected by the highly variable values of the GAG and HP content as a function of an inconsistent intra-individual and inter-individual appearance. Values obtained from the biochemical analysis showed a significant $(P<0.001)$ correlation between increasing ICRS grade and the content of GAG ( $\rho=0.521$ for grade $1 \sim 3$ up to $\rho=0.826$ for grade 1 2; Fig. 3a) and HP ( $\rho=0.645$ for grade 1 3 up to $\rho=0.852$ for grade 1 2; Fig. 3b). Furthermore, values showed minor regional distinctions with significant correlations between biochemical evaluation and the ICRS grade according to the femoral, medial and patellar compartment (Tab. 1). Values showed no correlation between the content of GAG and HP.

\section{Histological evaluation}

A total of 66 specimens were chosen for the histological examination representing the different stages of cartilage damage according to the ICRS classification (one sample of ICRS grade 0, 22 samples of ICRS grade 1, 23 samples of ICRS grade 2, and 20 samples of ICRS grade 3).

Values assessed from the histological analysis showed strong significant $(P<0.001)$ correlations between increasing ICRS grade and Mankin ( $\rho=$ $0.725)$ or Otte score $(\rho=0.736)$. Histological classification increased significantly with the macroscopical grade of cartilage damage classified by ICRS. Furthermore, values assessed from the histological analysis measured by the Score of Mankin were associated significantly with the grade of cartilage lesion according to the Score of Otte $(\rho=0.814)$. The results are summarized in Table 2 and visualized in Figure 4. The histological evaluation showed clear structural disorganization; fibrillation and cartilage defects increased significantly $(P<0.001)$ from ICRS grade 0 to 3 and approved the ICRS classification. Areas macroscopically classified as ICRS grade 1 showed fissures and cracks in the superficial and intermediate layer, hypercellularity and a decrease in safranin-O staining and were adjusted with $5.5 \pm 0.71$ points on the Mankin score and 1.19 \pm 0.15 on the Otte score. ICRS grade 2 with cellclustering of chondrocytes, deep cracks penetrating

Table 1. Biochemical results of the evaluation of GAG and HP content according to regional affiliations of the samples and the grade of cartilage lesion classified by ICRS.

\begin{tabular}{llllll}
\hline Localization & ICRS & General & Femoral & Tibial & Patellar \\
\hline GAG content in & 0 & $32.4(18,2)[6]$ & $24.5(17.1)[3]$ & $48.1(--)[3]$ & $--[0]$ \\
$\mu \mathrm{g} / \mathrm{mg}$ cartilage & 1 & $36.2(11.4)[334]$ & $37.6(14.2)[254]$ & $34.9(14.8)[58]$ & $34.1(8.1)[25]$ \\
& 2 & $34.4(10.9)[168]$ & $37.5(11.1)[96]$ & $33.2(12.9)[40]$ & $33.9(12.9)[32]$ \\
& 3 & $32.4(14.2)[105]$ & $39.5(15.6)[42]$ & $28.3(10.6)[42]$ & $26.1(9.3)[21]$ \\
\hline HP content in & 0 & $115.6(68.3)[6]$ & $139.5(77.1)[3]$ & $67.9(-)[3]$ & $--[0]$ \\
$\mu \mathrm{g} / \mathrm{mg}$ cartilage & 1 & $95.8(44.9)[334]$ & $95.7(47.3)[254]$ & $102.5(56,1)[58]$ & $99.9(25.7)[25]$ \\
& 2 & $80.9(42.4)[168]$ & $82.9(46.7)[96]$ & $79.4(35.3)[40]$ & $94.2(38.5)[32]$ \\
& 3 & $77.8(45.6)[105]$ & $76.2(46.1)[42]$ & $96.4(58.8)[42]$ & $72.1(46.5)[21]$
\end{tabular}

Data are expressed as means, standard deviation and total of samples. * Significant change at a level of $\mathrm{P}<0.05 ;{ }^{* *}$ significant change at a level of $P<0.001$

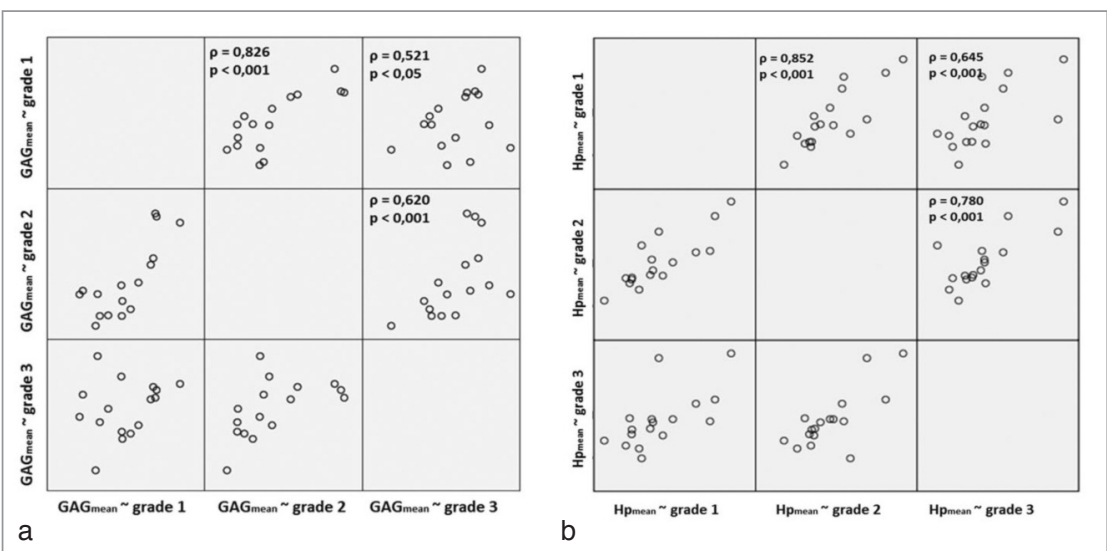

Figure 3. Dependence and correlation analysis between biochemical changes measured by the content of GAG (a) and HP (b), and the macroscopic grade of cartilage lesion classified by ICRS via bivariate correlations. 


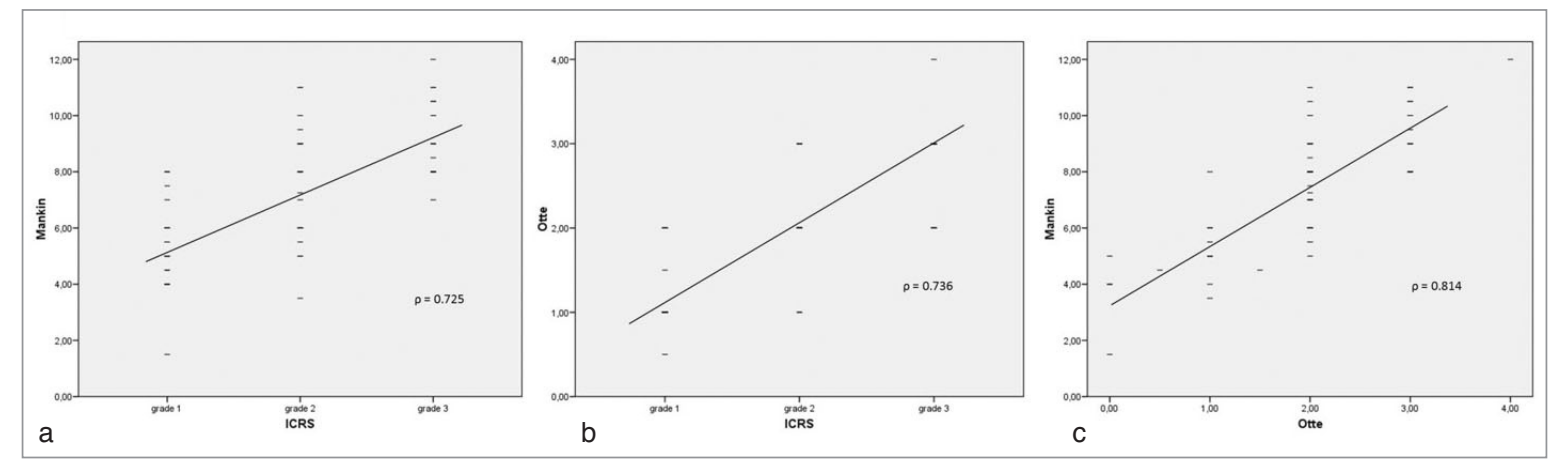

Figure 4. Dependence and correlation analysis between the macroscopical and histological grade of cartilage lesion classified by ICRS $(a, b)$, the Mankin $(a, c)$ and Otte $(b, c)$ score via bivariate correlations.

to the middle zone and a progressive decrease in safranin-O staining showed $7.51 \pm 0.43$ points on the Mankin score and $1.94 \pm 0.15$ on the Otte score. Cartilage changes associated with areas evaluated as ICRS grade 3 appeared with increased clefts and disruption of the matrix with cell-clustering, hypercellularity and an increase of the cartilage thickness. The Mankin score of this advanced stage of degeneration was $9.53 \pm 0.3$ points and the Otte score was $2.67 \pm 0.14$. Furthermore, values showed minor regional distinctions with significant correlations and differences between histological evaluation (Score of Mankin and Otte) and the ICRS grade according to the femoral, medial and patellar compartment (Tab. 2).

\section{Is there any correlation between biophotonical, bio- chemical and histological changes in OA?}

The NIRS CV correlated characteristically with the biochemical and histological changes. The NIRS CV decreases significantly $(P<0.05)$ with the grade of cartilage lesion according to the Mankin score $(\rho=$ 0.55) and Otte score $(\rho=0.5)$, shown in Figure 5. The NIRS CV was associated with changes in the content of GAG $(\rho=0.58 ; \mathrm{P}<0.05)$ and $\mathrm{HP}(\rho=$ $0.59 ; P<0.05)$. The NIRS CV decreased with the loss of GAG and HP content (Figs. 6, 7).

\section{Discussion}

The purpose of the current investigations was to prove whether NIRS is an objective and valid predictor identifying structural and biochemical changes in human cartilage of OA. To establish a diagnostic probe for the non-destructive evaluation of cartilaginous changes in composition and structure articular cartilage from human knee-joints with different degrees of OA was examined using biochemical, histological and biophysical techniques. Our data showed that biophotonical, histological, biochemical and visual characteristics of cartilage lesions correlated significant. Especially, NIR light absorption corresponded to the microscopical grade of cartilage lesions and to biochemical changes of cartilage structure and composition detected by the content of GAG and HP.

According to previous studies ${ }^{7}$, our results show that there is a strong correlation between the histological grading of the OA and the ICRS classification, and that the histological evaluation is one of the most reliable methods to determine the qualitative and quantitative extent of changes in cartilage. However, this method is limited to ex vivo examinations.

We are conscious that the arthroscopic grading of cartilage lesions due to the ICRS score as applied in this study is limited and has a poor inter-observer reliability, especially between intact cartilage (grade 0) and lesions that consist of softening of the cartilage

Table 2. Histological results of the evaluation of the Mankin score and Otte score according to regional affiliations of the samples and the grade of cartilage lesion classified by ICRS.

\begin{tabular}{llllll}
\hline Localization & ICRS & General & Femoral & Tibial & Patellar \\
\hline Mankin score & 0 & $5.5(--)[1]$ & $5.5(-)[1]$ & $--[0]$ & $--[0]$ \\
& 1 & $5.5(0.7)[22]^{* *}$ & $5.4(0.5)[21]^{* *}$ & $6.0(-)[1]^{* *}$ & $--[0]$ \\
& 2 & $7.5(0.4)[23]^{* *}$ & $7.4(0.6)[16]^{* *}$ & $9.0(0.2)[5]^{* *}$ & $4.5(1.4)[2]^{* *}$ \\
\hline Otte score & 3 & $9.5(0.3)[20]^{* *}$ & $9.9(0.3)[10]^{* *}$ & $9.0(0.3)[9]$ & $10.0(--)[1]^{* *}$ \\
& 0 & $1(--)[1]$ & $1(--)[1]$ & $--[0]$ & $--[0]$ \\
& 1 & $1.2(0.2)[22]^{* *}$ & $1.1(0.7)[21]^{* *}$ & $1(--)[1]^{* *}$ & $--[0]$ \\
& 2 & $1.9(0.2)[23]^{* *}$ & $1.9(0.7)[16]^{* *}$ & $2.4(0.5)[5]^{* *}$ & $1.5(0.7)[2]^{* *}$ \\
\hline
\end{tabular}

Data are expressed as means, standard deviation and total of samples. ${ }^{*}$ Significant change at a level of $\mathrm{P}<0.05 ;{ }^{* *}$ significant change at a level of $P<0.001$ 
Is there a correlation between biophotonical, biochemical, histological, and visual changes in the cartilage of osteoarthritic knee-joints?
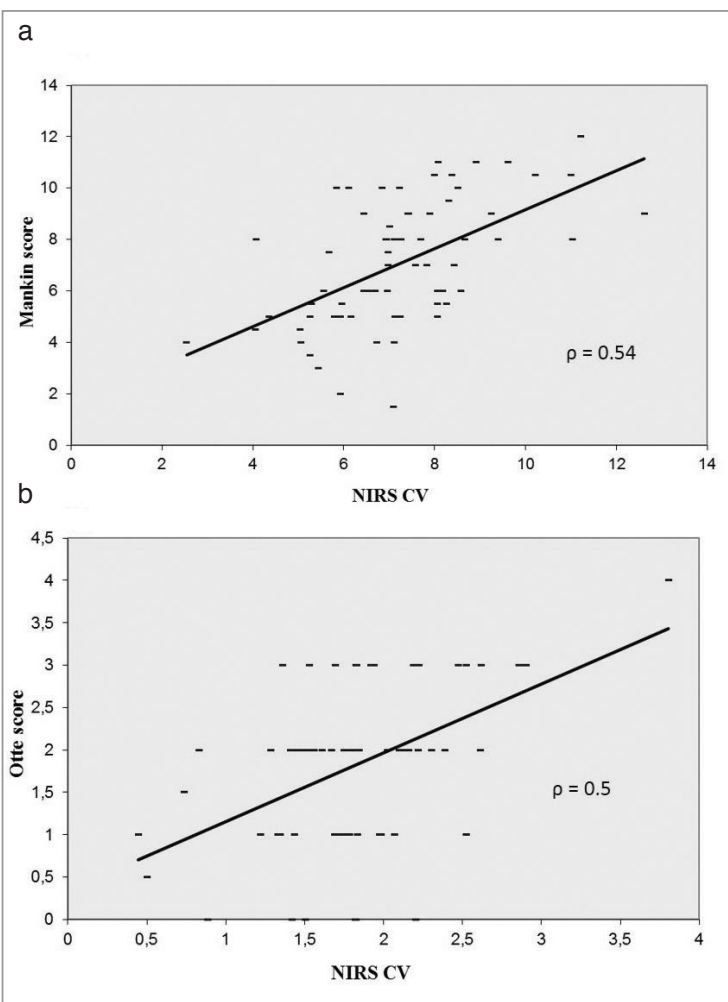

Figure 5. Dependence and correlation analysis between the NIRS and the grade of cartilage lesion classified by the Mankin (a) and Otte (b) score via bivariate correlations.

(grade 1), and differentiation between superficial and deep lesions ${ }^{21}$. All specimens, therefore, underwent a detailed histological scoring according to Mankin ${ }^{19}$ and $O t t e^{20}$. As has already been shown in previous studies, our results demonstrated that there is no histologically normal tissue in the OA joint ${ }^{22}$. The representative selection of histological samples $(n=66)$ shows no regions that are classified microscopically $(n=0)$ and macroscopically $(n=1)$ as normal cartilage.

It is a challenging task to establish a simple correlation between defined microscopical changes of cartilage structure and changes in biochemical composition. The biochemical composition of human cartilage (content of collagen and GAG) changes as a function of age, but also in OA. According to age, the collagen content of human cartilage is discussed controversially. Williamson et al. ${ }^{23}$ found that the collagen concentration in bovine cartilage increases during life as a function of age. In contrast, the GAG content remains unchanged or decreases slightly ${ }^{23}$. Other investigators found that proteoglycans are smaller and less functional with age ${ }^{8}$. Nevertheless, it is important to note that while changes in the composition and structure of the cartilage matrix take place inevitably, the development of $O A$ with aging is not a uniform process $^{24}$. This could be one explanation for our results showing no significant differences in matrix composition according to ICRS, and reflected the

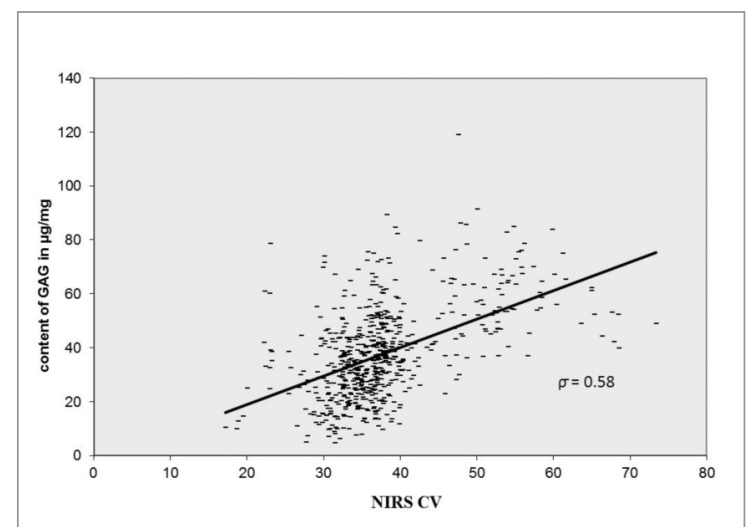

Figure 6. Dependence and correlation analysis between the NIRS and the content of GAG via bivariate correlations.

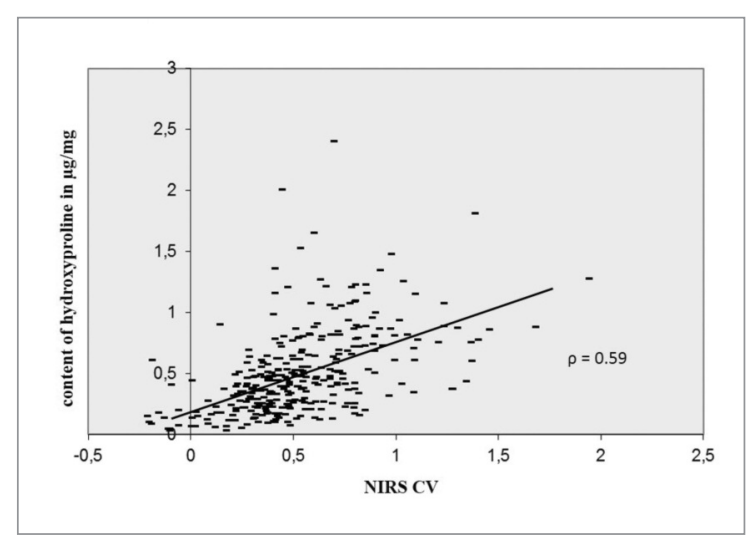

Figure 7. Dependence and correlation analysis between the NIRS and the content of HP via bivariate correlations.

high variability of the GAG and HP content as a function of an inconsistent intra-individual and inter-individual appearance. Furthermore, the mean age of our study patients is 68.71 years and structural changes observed during aging are not discriminated from disease-associated changes of articular cartilage.

Concerning the changes of the composition of cartilage in OA, some authors showed that the content of proteoglycan of the ECM is reduced in general 25,26 and that these proteoglycans exist in a non-aggregated and smaller form than in normal articular carti$\operatorname{lage}^{27}$. Aurich et al. ${ }^{5}$ were able to confirm the pronounced increase in collagen II synthesis and aggrecan turnover for the ankle-joint but not for the kneejoint. Early cartilage lesions in the knee-joint typically show an increase of collagen II cleavage.

Another result of the OA process and, additionally, a factor of aetiology of the disease is the deterioration in the mechanical properties of the cartilage. As a function of age, the elastic response of articular cartilage to loading forces decreases with decreasing content of $\mathrm{GAG}^{28}$. In a number of ex vivo studies in sheep femoral condyles, our group found an increased water content in combination with a decrease in mechanical stiffness, going along with an increasing degree of $\mathrm{OA}^{9,12}$. In contrast to the aforementioned studies in this setting, the biochemical analy- 
sis presented no significant differences in GAG and collagen content between intact cartilage (grade 0 ) and early stages of articular lesions (grade 1). Collagen and GAG content within the chondral defects decreased in tendency only in more severe lesions (grade 2 and grade $3)^{12}$. Marticke et al. ${ }^{29}$ found a significant correlation between the mechanical strength (Young's Modulus) of cartilage and different stages of degeneration quantified by the ICRS grade by investigating human knee-joint material. They also described a strong correlation between these mechanical alterations and biophotonical characteristics in the knee-joint cartilage analysed by the NIRS. In 2008, our group published the first results in evaluation of cartilage defects with NIRS from an ex vivo study in sheep femoral condyles ${ }^{12}$. The NIR absorption in ICRS grade 1 defects was significantly higher than in intact cartilage (grade 0). ICRS grade 2 specimens tended to a higher NIR absorption than the ICRS grade 1 specimen. A high correlation was found between the water content of the cartilage and NIR absorption, but no correlation was found between NIR absorption and collagen or GAG content ${ }^{12}$. In contrast, our results demonstrated moderate but significant associations between NIR light absorption and changes of cartilage structure and composition detected by the content of GAG and $\mathrm{HP}$, or the grade of cartilage lesion classified by the Mankin and Otte score. There are several lines of evidence indicating that the biochemical structural properties of cartilage are associated directly with the biophotonical properties ${ }^{14,30}$.

Our study has a number of limitations which may impair the interpretation of the study findings. Firstly, only cartilage of patients with severe OA needing TKR was available for this investigation. Secondly, our samples are acquired by a plurality of knees not delivering independent samples of each region (femoral, tibial and patellar) and ICRS grade. Thirdly, our investigations were performed in vivo. Tissue destructive methods were correlated with one non-destructive in vivo method describing changes of cartilage composition. Therefore, it is not remarkable that the correlations to biophotonical properties are only moderately represented compared to well-defined laboratory conditions or to quantitative assays. Nevertheless, we confirmed the correlation between cartilage degradation and changes of cartilage structure and composition with our investigation even if femoral or tibial cartilage was separately surveyed, and we established NIRS for the detection of biochemical and microscopical changes of cartilage composition and lesion.

Furthermore the quantitative differentiation of early-stage cartilage defects (grade 1) and intact cartilage (grade 0) is a rather difficult task and is, in part, still unsolved ${ }^{7,18}$. Moreover, even the differentiation between grade 2 and 3 cartilage defects is difficult concerning the depth of the lesion $^{18}$. The intraoperative visual and tactile classification is very subjective, based on the individual experience of the investigator and sometimes hardly reproducible ${ }^{31}$, and has a poor interobserver agreement ${ }^{21}$. Thus, a more precise description of the location, depth and size of the cartilage lesion adjacent to unchanged cartilage is mandatory for diagnosis and therapy ${ }^{18}$. Iden- tifying early OA changes in cartilage, as well as monitoring different cartilage repair and regeneration therapies, requires reliable imaging technology. Recent clinical studies have shown that NIRS is a proven sensitive and accurate diagnostic method for early stages of cartilage damage and initial $\mathrm{OA}^{32}$. In addition, NIRS proved to provide a good interobserver correlation ${ }^{21}$. In another study with 21 patients with knee pain lasting for at least half a year without any trauma of the knee, magnetic resonance imaging and arthroscopy had a rather low predictive value for early-stage cartilage lesions. Only NIRS found a correlation to the complaints (estimated by the KOOS) in these patients ${ }^{13}$.

Nevertheless, since water content, mechanical properties of cartilage and cartilage damage (ICRS) can already be predicted employing the NIRS technique with rather good accuracy, our data suggest that it is not possible to explain the underlying biochemical changes in OA with this technique up to now. Therefore, it is necessary to develop different artificial cartilage models to calibrate NIRS to define chemical changes in these settings under laboratory conditions ${ }^{30}$.

NIR is a viable option for clinical characterisation of articular cartilage integrity ${ }^{33}$, but more work is still needed in order to bring NIR application to the advanced state of IR characterisation of articular cartilage.

Summarized, these data show that changes in the composition and structure of cartilage due to the pathogenesis of OA influence the optical properties that can be measured by NIRS. The quality of this technical evaluation, nevertheless, cannot compete with the laboratory testing (biochemical and histological) and results that are available with in vitro optical measurements. This is due to the setting where the biophotonical testing was performed in an in vivo-like measurement. The ease of use during arthroscopy, the prompt response and the non-destructive nature of NIRS make it a promising additional diagnostic technique in $\mathrm{OA}$.

\section{Acknowledgements}

This work has been supported in part by the German Working Compensation (Berufsgenossenschaft für die Bauwirtschaft) in Berlin and by the Thüringer Aufbaubank in Erfurt. Grateful thanks are given to Birgit Lemser for excellent assistance in laboratory work.

\section{Competing interest statement}

G. O. Hofmann and H. P. W. Plettenberg hold shares in arthrospec $\mathrm{GmbH}$, Jena.

\section{References}

1. Hunter DJ, Felson DT. Osteoarthritis. BMJ 2006; 332(7542):639-642.

2. Abramson SB, Attur M. Developments in the scientific understanding of osteoarthritis. Arthritis Res Ther 2009; 11. 
Is there a correlation between biophotonical, biochemical, histological, and visual changes in the cartilage of osteoarthritic knee-joints?

3. Poole AR. Cartilage in health and disease. Koopman WJ ed Arthritis and Allied Conditions: A Textbook of Rheumatology. Philadelphia; Lippincott Williams and Wilkins 2005; 223-269.

4. Squires GR, Okouneff S, lonescu M, Poole AR. The pathobiology of focal lesion development in aging human articular cartilage and molecular matrix changes characteristic of osteoarthritis. Arthritis rheum 2003; 48(5):1261-1270.

5. Aurich M, Squires GR, Reiner A, et al. Differential matrix degradation and turnover in early cartilage lesions of human knee and ankle joints. Arthritis rheum 2005; 52(1):112-119.

6. Bobinac D, Spanjol J, Zoricic S, Maric I. Changes in articular cartilage and subchondral bone histomorphometry in osteoarthritic knee joints in humans. Bone 2003; 32(3):284-290.

7. Kleemann RU, Krocker D, Cedraro A, Tuischer J, Duda GN. Altered cartilage mechanics and histology in knee osteoarthritis: relation to clinical assessment (ICRS Grade). Osteoarthritis cartilage 2005; 13(11):958-963.

8. Buckwalter JA, Saltzman C, Brown T. The impact of osteoarthritis: implications for research. Clin Orthop Relat Res 2004; (427 Suppl):S6-15.

9. Spahn G, Plettenberg $\mathrm{H}$, Nagel H, et al. Karl Fischer titration and coulometry for measurement of water content in small cartilage specimens. Biomed Tech (Berl) 2006; 51(5-6):355359.

10. Jöbsis FF. Noninvasive, infrared monitoring of cerebral and myocardial oxygen sufficiency and circulatory parameters. Science 1977;198:1264-1267.

11. Wolf M, Ferrari M, Quaresima V. Progress of near-infrared spectroscopy and topography for brain and muscle clinical applications. J Biomed Opt 2007; 12(6):062104.

12. Spahn G, Plettenberg $H$, Nagel $H$, et al. Evaluation of cartilage defects with near-infrared spectroscopy (NIR): an ex vivo study. Med Eng Phys 2008;30(3):285-292.

13. Hofmann GO, Marticke J, Grossstuck R, et al. Detection and evaluation of initial cartilage pathology in man: A comparison between MRT, arthroscopy and near-infrared spectroscopy (NIR) in their relation to initial knee pain. Pathophysiology 2010; 17(1):1-8.

14. Johansson A, Kuiper JH, Sundqvist T, et al. Spectroscopic measurement of cartilage thickness in arthroscopy: ex vivo validation in human knee condyles. Arthroscopy 2012; 28:1513-1523.

15. Roos EM, Lohmander LS. The knee injury and osteoarthritis outcome score (KOOS): from joint injury to osteoarthritis. Health Qual Life Outcomes Health Qual Life 2003; 64772.

16. Roos EM, Roos HP, Lohmander LS, Ekdahl C, Beynnon BD. Knee injury, osteoarthritis outcome score (KOOS) - development of a self-administered outcome measure. J Orthop Sports Phys Ther 1998; 28(2):88-96.

17. Kellgren JH, Lawrence JS. Radiological assessment of osteoarthritis. Ann Rheum Dis 1957; 16(4):494-502.

18. Brittberg M, Winalski CS. Evaluation of cartilage injuries and repair. J Bone Joint Surg Am 2003; 85-A Suppl 2:58-69.
19. Mankin HJ, Dorfman H, Lippiello L, Zarins A. Biochemical and metabolic abnormalities in articular cartilage from osteoarthritic human hips. II. Correlation of morphology with biochemical and metabolic data. J Bone Joint Surg Am 1971; 53(3):523-537.

20. Otte P. Degeneration of joint cartilage. Clinical and radiological aspects. Munch Med Wochenschr 1968; 110(46):2677-2683.

21. Spahn G, Klinger HM, Baums M, et al. Near-infrared spectroscopy for arthroscopic evaluation of cartilage lesions: results of a blinded, prospective, interobserver study. Am J Sports Med 2010; 38(12):2516-2521.

22. Heinegard D, Lidgren L, Saxne T. Recent developments and future research in the bone and joint decade 2000-2010. Bull World Health Organ 2003; 81(9):686-688.

23. Williamson AK, Chen AC, Masuda K, Thonar EJ, Sah RL. Tensile mechanical properties of bovine articular cartilage: variations with growth and relationships to collagen network components. J Orthop Res 2003; 21(5):872-880.

24. Carrington JL. Aging bone and cartilage: cross-cutting issues. Biochem Biophys Res Commun 2005; 328(3):700-708.

25. Anderson CE, Ludowieg J, Harper HA, Engleman EP. The Composition of the Organic Component of Human Articular Cartilage. Relationship to Age and Degenerative Joint Disease. J Bone Joint Surg Am 1964; 46:1176-1183.

26. Mankin HJ, Lippiello L. The glycosaminoglycans of normal and arthritic cartilage. J Clin 1971; 50(8):1712-1719.

27. Brandt KD, Palmoski M. Organization of ground substance proteoglycans in normal and osteoarthritic knee cartilage. Arthritis Rheum 1976; 19(2):209-215.

28. Kempson GE, Muir H, Swanson SA, Freeman MA. Correlations between stiffness and the chemical constituents of cartilage on the human femoral head. Biochim Biophys Acta 1970; 215(1):70-77.

29. Marticke JK, Hosselbarth A, Hoffmeier KL, et al. How do visual, spectroscopic and biomechanical changes of cartilage correlate in osteoarthritic knee joints? Clin Biomech 2010; 25(4):332-340.

30. Zierbock S, Plettenberg H, Schmitt M, Liebold S, Hoffmann M, Popp J. NIR spectroscopic analyses of chemical osteoarthritic cartilage models. Biomed Tech 2012.

31. Spahn G, Klinger HM, Baums M, Pinkepank U, Hofmann GO. Reliability in arthroscopic grading of cartilage lesions: results of a prospective blinded study for evaluation of inter-observer reliability. Arch Orthop Trauma Surg 2011; 131(3):377-381.

32. Spahn G, Wittig R, Kahl E, Klinger HM, Muckley T, Hofmann GO. Evaluation of cartilage defects in the knee: validity of clinical, magnetic-resonance-imaging and radiological findings compared with arthroscopy. Unfallchirurg 2007; 110(5):414-424.

33. Afara I, Prasadam I, Crawford R, Xiao Y, Oloyede A. Nondestructive evaluation of articular cartilage defects using nearinfrared (NIR) spectroscopy in osteoarthritic rat models and its direct relation to Mankin score. Osteoarthritis 2012; 20(11):1367-1373. 